\title{
Correlation Between Apelin and Collateral Circulation in Patients with Middle Cerebral Artery Occlusion and Moyamoya Disease
}

\author{
Hanlin Wu (D) ${ }^{1, *}$ \\ Chengyu $\mathrm{Xia}^{2, *}$ \\ Rui $\mathrm{Li}^{3}$ \\ Chunrong $\mathrm{Tao}^{3}$ \\ Qiqiang Tang ${ }^{3}$ \\ Wei Hu'
}

'Stroke Center \& Department of Neurology, The First Affiliated Hospital of USTC, Division of Life Sciences and Medicine, University of Science and Technology of China, Hefei, Anhui, People's Republic of China; ${ }^{2}$ Department of Neurosurgery, The First Affiliated Hospital of USTC, Provincial Hospital Affiliated to Anhui Medical University, Hefei, Anhui, People's Republic of China; ${ }^{3}$ Department of Neurology, The First Affiliated Hospital of USTC, Provincial Hospital Affiliated to Anhui Medical University, Hefei, Anhui, People's Republic of China

*These authors contributed equally to this work
Correspondence: Wei Hu; Qiqiang Tang Email andinghu@126.com; tqq1995@126.com
Background and Objectives: Moyamoya disease (MMD) is a unique cerebrovascular occlusive disease with abnormal vascular hyperplasia, which causes cerebrovascular accidents like intracranial arteriosclerosis. This study aimed to explore whether plasma apelin levels are related to good collateral circulation in ischemic diseases, which may be higher in patients with MMD than middle cerebral artery (MCA) occlusion or healthy controls, and may have a connection with the MMD grades.

Methods: We recruited $68 \mathrm{MMD}$ patients and $25 \mathrm{MCA}$ occlusion patients diagnosed by angiography, including 29 patients without cerebrovascular problems as controls. We examined the plasma apelin, serum nitric oxide (NO), and vascular endothelial growth factor (VEGF) levels of all subjects by ELISA kit. We compared the relationship between apelin, NO, and VEGF in the blood of three groups, to explore the relationship. We also investigated whether the plasma apelin-13, apelin-17, and apelin-36 levels correlate with the MMD classification.

Results: Univariate analyses indicated that the MMD group had the higher plasma apelin13, apelin-17, apelin-36, and serum NO levels than the MCA occlusion and healthy control groups. Binary logistic regression analyses further showed that the apelin-13 level was substantially higher in MMD patients than in MCA occlusion patients. Patients with MMD were significantly younger than patients with MCA occlusion by their mean ages. Linear regression analyses were performed to compare apelin levels between different grades of the patients with MMD. Apelin-13, apelin-17, and apelin-36 levels increased with the gradual increase of compensation grades level independent of NO and VEGF. Apelin-13 and apelin36 showed a positive effect on the compensation scores in MMD.

Conclusion: Our study demonstrated that apelin-13 was significantly increased in patients with MMD than patients with MCA occlusion independent of NO and VEGF. Moreover, plasma apelin-13, apelin-17, and apelin-36 levels increase with the grades of MMD.

Keywords: apelin, collateral circulation, moyamoya disease, MMD, middle cerebral artery occlusion, MCA occlusion

\section{Introduction}

Moyamoya disease (MMD) is a rare cerebral vasculature disease with longstanding and progressive occlusion of large intracranial arteries. ${ }^{1}$ It is characterized by chronic progressive nonatherosclerotic stenosis or occlusion of the bilateral internal carotid artery (ICA), anterior cerebral artery (ACA) and middle cerebral artery (MCA), and secondary formation of an abnormal vascular network at the base of the skull. MMD usually occurs in the East Asian population, mostly 
affecting women than men. Epidemiologically, the incidence of MMD is 0.94 and 2.3 per 100,000 individuals in Japan and South Korea. ${ }^{2}$ A narrow proportion of patients are familial, but most cases of MMD are sporadic. However, the exact etiology is still not well established. ${ }^{3}$ MMD has a bimodal characteristic in East Asia, with a high incidence in children around 10 years old and adults around 40 years old, manifested as hemorrhagic and ischemic. ${ }^{1}$ The clinical characteristics of ischemic type are similar to those of arteriosclerotic ischemic stroke, patients with MMD or intracranial atherosclerotic disease experience similar cerebral ischemic events. Ischemic stroke is a leading cause of death worldwide, ${ }^{4}$ with a higher incidence rate of disability after stroke and poor prognosis. Previous studies reported that a rich supply of blood through collateral circulation could reduce expansion of the ischemic area and improve clinical outcomes after the proximal artery occlusion. ${ }^{5}$ The development of moyamoya vessels or collateral vessels provides an alternative route for compromised cerebral perfusion. ${ }^{6,7}$ However, such compensation is often insufficient or unbalanced, resulting in various ischemic or hemorrhagic strokes. ${ }^{8}$ A previous study reported that compared with patients diagnosed with chronic cerebral ischemia, patients with MMD have a higher potential for angiogenesis as well as better clinical prognoses and outcomes. ${ }^{9}$ Stroke with MCA occlusion caused by intracranial cerebral atherosclerotic thrombosis is more common in Asians. ${ }^{10}$ Most researchers believe that establishing collateral circulation requires a combination of many factors, such as apelin or vascular growth factors, etc. ${ }^{11}$

Apelin is the endogenous ligand of the G proteincoupled receptor APJ. Apelin and APJ receptors are widely expressed in humans, especially the central nervous system and in the periphery (heart, brain, lung, kidney, adipose tissue, limbs, retina, liver, skin). ${ }^{12}$ The previous report indicates that the Apelin/APJ system plays an important role in pathological angiogenesis, with the progressive development of new blood branches from preexisting via sprouting. ${ }^{11}$ Hiroyasu et $\mathrm{al}^{13}$ proposed that APJ is expressed in endothelial cells at the edge of vessels during early embryogenesis, in combination with VEGF, which can induce the proliferation and assembly of endothelial cells. Some studies supported that Apelin combined with other angiogenic factors plays an important role in angiogenesis. ${ }^{14,15}$ However, the role of the Apelin/APJ system in the MMD progression is rarely investigated.
To sum up, we assumed that the apelin level might indicate the formation of neovascularization, the abundance of collateral circulation, and the level can serve as differentiation for MMD from MCA occlusion cooperatively with nitric oxide (NO) and VEGF. Moreover, the apelin plasma levels may change along with grades of collateral vessels compensatory in patients with MMD. In the present study, we attempted to explore these assumptions by investigating the association between plasma apelin-13, apelin-17, apelin-36, and serum NO, VEGF levels in patients, by comparing the apelin levels in MMD and MCA occlusion to find out the difference and summarize the possible mechanism. Furthermore, we aimed to evaluate whether there is a linear relationship between Apelin and collateral circulation compensation.

\section{Methods}

\section{Study Design}

Informed consent of publishing information/images was obtained from all participants or their family members for the present study. All study procedures were approved by the Ethics Committee of the Affiliated Provincial Hospital of Anhui Medical University and conducted according to the Helsinki Declaration (1975 and subsequent revisions).

\section{Participants}

A total of 68 patients with MMD, 25 patients with MCA occlusion, and 29 stroke-free individuals (controls) were enrolled over 2 years (November 2018-January 2020) for the current study. All subjects underwent computed tomography $(\mathrm{CT})[\mathrm{n}=21]$ or magnetic resonance imaging (MRI) [ $\mathrm{n}=101]$, which detected the occurrence of ischemic or hemorrhagic stroke. All patients with intracranial arterial occlusion have undergone computed tomographic angiography (CTA) [n=82] or digital subtraction angiography (DSA) $[n=78]$ to evaluate blood vessel condition, which was completed within 1-5 days after admission.

The diagnostic criteria of MMD stated the guidelines for diagnosis and treatment of MMD (spontaneous occlusion of the circle of Willis) published by Japanese scientists in $2012 .{ }^{16}$ Cerebral angiography is essential for the diagnosis, and MRI and MRA results can also be used to diagnose unfavorable conditions, the former is more commonly used in scientific research. The results of cerebral angiography in this study must exhibit the following points: (1) stenosis or occlusion of the intracranial artery; 
(2) abnormal vascular networks in the vicinity of the occlusive or stenotic lesions in the arterial phase; and (3) bilaterality of findings (1) and (2). MMD is a cerebrovascular disease of unknown etiology, if vascular disease is related to the following basic diseases, it should be excluded: (i) atherosclerosis; (ii) autoimmune disease; (iii) meningitis; (iv) brain tumors; (v) Down's syndrome; (vi) von Recklinghausen's disease; (vii) head injury; and (viii) cerebrovascular lesions after head irradiation, etc.

The diagnosis of MCA occlusion was based on ischemic stroke, ${ }^{17}$ and the DSA or CTA examination showed stenosis and occlusion of the middle cerebral artery. Patients with the following manifestations are of great value in diagnosis: (1) multiple transient ischemic attacks (TIA) occurred in the history, mostly in the blood supply area of a middle cerebral artery; (2) aphasia, neglect, motor function impairment or cerebellum, brain stem damage; (3) carotid auscultation with murmur, pulse weakening, bilateral blood pressure asymmetry; (4) carotid stethoscope with abnormal blood pressure, brain CT or MRI examination can find cerebral cortex or cerebellum lesions, or subcortical, brainstem lesions diameter $>1.5 \mathrm{~cm}$, maybe the potential of large artery atherosclerosis caused by ischemic stroke; (5) color ultrasound, transcranial Doppler ultrasound (TCD), MRA or digital subtraction angiography (DSA) examination can find related middle cerebral artery and its branches stenosis degree $>50 \%$; and (6) stroke caused by cardiac embolism should be excluded. CTA or DSA were performed in all patients with MCA occlusion, the collateral circulation of MCA occlusion patients was graded by ASITN/SIR score system based on the images. ${ }^{18}$

Patients hospitalized during the same period, with a complaint of dizziness and headache, and CT/MRI examinations showing no apparent abnormalities served as a control in the current study. Subjects were excluded from enrolment for contraindication to iodinated contrast agent administration (history of contrast agent allergy, pregnancy, congestive heart failure, and renal insufficiency), previous revascularization history (cerebral artery bypass grafting or cerebral artery intervention), evidence of ongoing infection or inflammation or autoimmune diseases, chronic obstructive pulmonary disease, previous diagnosis of malignancy, diabetes with a history of insulin usage, and juvenile patients with MMD. ${ }^{19}$

\section{The Grading System of MMD}

Patients with MMD were further classified according to the new classification standard described previously. The grading score was obtained based on the collateral circulation and ranged from 1 to 12 . The scoring system was mainly divided into two parts according to the distribution of intracranial vessels. The leptomeningeal system includes the following 3 parts of the collateral networks according to the anatomy extent of pial collateral blood from the posterior cerebral artery (PCA) territory to the anterior cerebral artery (ACA) territory and the MCA territory on the delayed venous phase. The perforators reflected the supply of the anterior collateral circulation and internal cerebral artery Thus, we defined the expansion of this collateral route using the Suzuki stage as follows: scores of 6 to 1 corresponding to Suzuki stages 1 to $6 .{ }^{19,20}$ Further details are available online [https:// www.ahajournals.org/doi/suppl/10.1161/STROKEAHA. 119.024487]. The 3 collateral circulation status grades in MMD were defined as follows: scores of 1 to 4 were defined as a poor collateral status (grade I), scores of 5 to 8 were defined as a fair collateral status (grade II), and scores of 9 to 12 points were defined as a good collateral status (grade III).

All angiography images were reviewed by two experts who were blinded to the patient details. Any differences in their observations were resolved by consensus.

\section{Analysis of Lipids, Nitric Oxide, and Apelin}

Blood samples were collected on the day after admission after fasting for $12 \mathrm{~h}$. For apelin, $5 \mathrm{~mL}$ blood samples were collected in tubes containing EDTA-K2. Immediately the following centrifugation at $3000 \mathrm{rpm}$ for $10 \mathrm{mins}$, the plasma samples were preserved at $-80{ }^{\circ} \mathrm{C}$ for further analysis. Apelin-13, apelin-17, and apelin-36 levels were assayed using commercially available enzyme immunoassay kits (Novus Biologicals, USA). For NO and VEGF, blood $(5 \mathrm{~mL})$ was collected and centrifuged at $3000 \mathrm{rpm}$ for 10 mins. The serum samples were separated and stored at $-80{ }^{\circ} \mathrm{C}$. The serum NO level was determined using a nitrate/nitrite colorimetric assay kit (Cayman Chemical, MI, USA). The serum VEGF levels were tested by Enzyme-Linked Immunosorbent Assay kits (ELISA, Biogems, USA). Given the lack of sample size, we were unable to detect serum VEGF in the control group. 
Different researchers reevaluated blood pressure, BMI, and biochemical parameters to ensure data accuracy.

\section{Statistical Analysis}

Continuous variables conforming to the normal distribution are presented as the mean \pm standard deviation (SD), and those not conforming to the normal distribution are represented by median $\pm \mathrm{IQR}$ (interquartile range); categorical variables are presented as percentages. Independent sample $t$-test was used for normal distribution variables, and the rank-sum test was used for variables that did not meet the normal distribution. Differences in categorical variables among the groups were examined using the chisquare test or Fisher test. Comparisons of continuous variables were analyzed with one-way analysis of variance, Mann-Whitney $U$, or Kruskal-Wallis tests. Pearson analysis was conducted to find a correlation between factors. Regression analysis was constructed to establish the equation of apelin and other parameters for the differentiation of the group with MMD and MCA occlusion (binary logistic and linear regression). Factors with values of $P<0.1$ were tested in multivariable binary logistic regression, which was adjusted by confounding factors, the optimal cut-off values of apelin were figured out in the binary logistic regression model. Considering that the number of patients in the MMD and MCA occlusion groups was significantly different, propensity matching was used, 18 matched subjects were obtained after adjusting the age, gender, and BMI. The Kruskal-Wallis test and/or $t$-test were conducted to compare the levels of Apelin factors between the two groups.

According to the condition of collateral circulation and the progress of the disease course, all patients were divided into three groups (as described earlier). Linear regression was conducted to compare apelin-13, apelin17 , and apelin-36 level of three groups and find the law of the correlation between apelin levels and the course of MMD. All analyses were performed using Statistical Package for Social Sciences software (SPSS 20.0). Figures were drawn by GraphPad Prism 8.

\section{Results}

\section{Baseline Characteristics}

The current study recruited 68 patients with MMD, 25 patients with MCA occlusion, and 29 healthy participants. Among the 68 MMD patients, $34(50 \%)$ of them with MCA occlusion, $26(38.2 \%)$ were men, the ratio was significantly higher than the other two groups. Five (7.4\%) had a history of smoking and drinking, 7 (10.3\%) accepted aspirin and atorvastatin treatment, $2(2.9 \%)$ as well as clopidogrel treatment, and the withdrawal time was more than half a year. The average age was $46.66 \pm 9.65$ years. There were 24 patients with hemorrhagic MMD, the rest of them were diagnosed as ischemic MMD. Thirtythree $(48.5 \%)$ of the patients with MMD had a history of stroke, 7 (28\%) of the patients with MCA occlusion had a prior ischemic stroke. The average time from the onset of symptoms to angiographic examination in the MMD group was $11.02 \pm 17.89$ months, and the average time in the MCA occlusion group was $2.42 \pm 6.28$ months. Among the 25 patients with MCA occlusion, 19 (76\%) were men, $11(44 \%)$ had a history of smoking, $8(32 \%)$ had a history of drinking, $2(8 \%)$ accepted aspirin, atorvastatin and clopidogrel treatment. The average age was $53.24 \pm 12.16$ years, which was higher in the three groups. Among 29 healthy controls, $15(51.7 \%)$ were men, and the average age was $51 \pm 11.53$ years.

The general characteristics, including vascular risk factors and laboratory findings, are summarized in Table 1. The mean age, ratio of males to females, diastolic blood pressure (DBP), and the NHISS scores were significantly higher in the MCA occlusion group than the other two groups. The relationship of plasma apelin-13, apelin-17, apelin-36, and serum NO, VEGF levels between the three groups are shown in Figure 1.

\section{Differentiating Factors Between MMD and MCA Occlusion}

The levels of apelin-13, apelin-17, and apelin-36 were significantly higher in patients in the MMD group than patients with MCA occlusion, and the results in both groups were significantly higher than the control group (Figure 1). For serum NO and VEGF levels, the patients with MMD and MCA occlusion were significantly higher than controls, no statistical difference was observed between the two. The NHISS score in the MCA occlusion group is significantly higher than in patients with MMD. The results of binary logistics regression adjusted by BMI, sex, cigarette smoking, and alcohol consumption demonstrated that apelin-13 and age were significantly increased in patients with MMD than in the MCA occlusion group (Table 2). To further identify MMD from MCA occlusion, receiver operating characteristic (ROC) curve analyses indicated that the apelin-13 level of $196.79 \mathrm{ng} / \mathrm{mL}$ had 
Table I Univariate Analysis of Control, MMD, and MCA Occlusion Groups

\begin{tabular}{|c|c|c|c|c|}
\hline Parameters & Controls (29) & MMD Group (68) & MCA Occlusion Group (25) & $P$-value \\
\hline Age, years ${ }^{b}$ & $51.00 \pm 10.50$ & $46.66 \pm 9.65$ & $53.24 \pm 12.16$ & 0.009 \\
\hline Sex, male $(n, \%)^{c}$ & $15(51.7)$ & $26(38.2)$ & $19(76)$ & 0.005 \\
\hline BMI, $\mathrm{kg} / \mathrm{m}^{2 \mathrm{a}}$ & $24.44 \pm 3.39$ & $24.93 \pm 3.10$ & $25.64 \pm 3.32$ & 0.476 \\
\hline $\mathrm{SBP}(\mathrm{mmHg})^{\mathrm{a}}$ & $133.10 \pm 18.53$ & $136.25 \pm 16.58$ & $|44.84 \pm| 7.94$ & 0.050 \\
\hline $\mathrm{DBP}(\mathrm{mmHg})^{\mathrm{a}}$ & $80.38 \pm 9.09$ & $84.97 \pm 9.25$ & $88.48 \pm 9.58$ & 0.006 \\
\hline Smoke $(\mathrm{n}, \%)^{\mathrm{c}}$ & $7(24.1)$ & $5(7.4)$ & II (44) & $<0.001$ \\
\hline Drunk $(\mathrm{n}, \%)^{\mathrm{c}}$ & $6(20.7)$ & $5(7.4)$ & $8(32)$ & 0.010 \\
\hline Stroke type $(n, \% \text {, ischemic })^{c}$ & 0 & $44(64.7)$ & $25(100)$ & 0.001 \\
\hline Stroke history $(\mathrm{n}, \%)^{\mathrm{c}}$ & 0 & $33(48.5)$ & $7(28)$ & 0.078 \\
\hline Total cholesterol $(\mathrm{mg} / \mathrm{dL})^{\mathrm{a}}$ & $4.33 \pm 0.72$ & $4.07 \pm 1.09$ & $3.77 \pm|.3|$ & 0.077 \\
\hline Triglyceride $(\mathrm{mg} / \mathrm{dL})^{\mathrm{a}}$ & $2.06 \pm 1.80$ & $1.27 \pm 0.50$ & $1.27 \pm 0.96$ & 0.194 \\
\hline LDL-cholesterol $(\mathrm{mg} / \mathrm{dL})^{\mathrm{a}}$ & $2.59 \pm 0.76$ & $2.42 \pm 0.82$ & $1.99 \pm 1.00$ & 0.285 \\
\hline Lipoprotein a $(\mathrm{mmol} / \mathrm{L})^{\mathrm{a}}$ & $279.26 \pm 249.06$ & $270.84 \pm 251.37$ & $356.91 \pm 227.37$ & 0.165 \\
\hline Blood glucose $(\mathrm{mmol} / \mathrm{L})^{\mathrm{a}}$ & $5.20 \pm 0.97$ & $4.79 \pm 0.70$ & $5.83 \pm 1.23$ & 0.002 \\
\hline Homocysteine $(\mu \mathrm{mol} / \mathrm{L})^{\mathrm{a}}$ & $12.17 \pm 5.23$ & $10.00 \pm 4.00$ & $12.25 \pm 5.93$ & 0.911 \\
\hline Apelin-13 (pg/mL) & $32.15 \pm 28.14$ & $271.15 \pm 137.85$ & $155.18 \pm 105.73$ & 0.000 \\
\hline Apelin- 17 (ng/dL) ${ }^{b}$ & $60.99 \pm 63.13$ & $196.78 \pm 75.82$ & $139.04 \pm 60.74$ & 0.000 \\
\hline Apelin-36 (ng/dL) ${ }^{b}$ & $354.05 \pm 244.14$ & $957.80 \pm 481.28$ & $727.11 \pm 250.39$ & 0.000 \\
\hline $\mathrm{NO}(\mathrm{nmol} / \mathrm{L})^{\mathrm{b}}$ & $41.02 \pm 36.08$ & $113.21 \pm 135.18$ & $112.59 \pm 105.53$ & 0.000 \\
\hline VEGF $(p g / m L)^{b}$ & & $195.48 \pm 405.12$ & $166.81 \pm 161.39$ & 0.840 \\
\hline Aspirin treatment $(\%)^{c}$ & I (3.4) & $7(10.3)$ & $2(8)$ & 0.531 \\
\hline 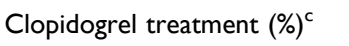 & $0(0)$ & $2(2.9)$ & $2(8)$ & 0.251 \\
\hline Atorvastatin $(\%)^{c}$ & I (3.4) & $7(10.3)$ & $2(8)$ & 0.531 \\
\hline NHISS ${ }^{\mathrm{b}}$ & $0.14 \pm 0.58$ & $1.40 \pm 3.6 \mathrm{I}$ & $4.40 \pm 3.74$ & 0.000 \\
\hline
\end{tabular}

Notes: Continuous data conform to normal distribution are presented with mean $\pm \mathrm{SD}$ marked as "a”, and abnormal distribution data are presented as median $\pm \mathrm{IQR}$ (interquartile range), which is marked as "b", the counting data of said frequency and percentage marked as "c".

BMI, body mass index; SBP, systolic blood pressure; DBP, diastolic blood pressure; NO, nitric oxide; MMD, moyamoya disease; MCA, middle cerebral artery; NHISS, National Institutes of Health Stroke Scale.

a $72 \%$ sensitivity and an $84 \%$ specificity (Figure $2 \mathrm{~A}$ ) and 57.5 years old had a $44 \%$ sensitivity and an $88.2 \%$ specificity (Figure 2B), which can identify MMD from MCA occlusion.

After propensity matching, we conducted an intergroup test on 18 pairs of samples corrected for age, gender and BMI, and found that plasma apelin-13 and apelin-17 levels were still significantly higher in the MMD group (2797.02 \pm 308.59 ; 198.86 \pm 16.57$)$ than the MCA occlusion group (1675.91 $\pm 290.12 ; 137.28 \pm 14.51)$ (Supplementary files).

\section{Plasma Apelin Levels in Patients with Different Grades of MMD}

According to a new grading system, all patients with MMD were classified into three grades, which was published in Stroke in $2019 .^{20}$ The grades were correlated with the abundance of vessels in the medial cranial branches.
MMD patients were divided into three groups according to individual score. The compensation is from poor to good (numbers: Grade I: 11, Grade II: 27, Grade III: 30). Linear regression analyses were performed to compare the apelin levels between different grades of MMD patients. A trend of gradual increases with the rise of the grades was demonstrated (Figure 3).

The images of DSA in patients with MMD are shown in Figure 4. The compensation gradually improves from left to right (from Grade I to Grade III).

\section{Discussion}

We found significantly increased plasma apelin-13, apelin17, and apelin-36 levels in patients with MMD compared to patients with MCA occlusion and healthy controls, especially those diagnosed with MCA occlusion. Apelin13 and age are significant factors in differentiating MMD 

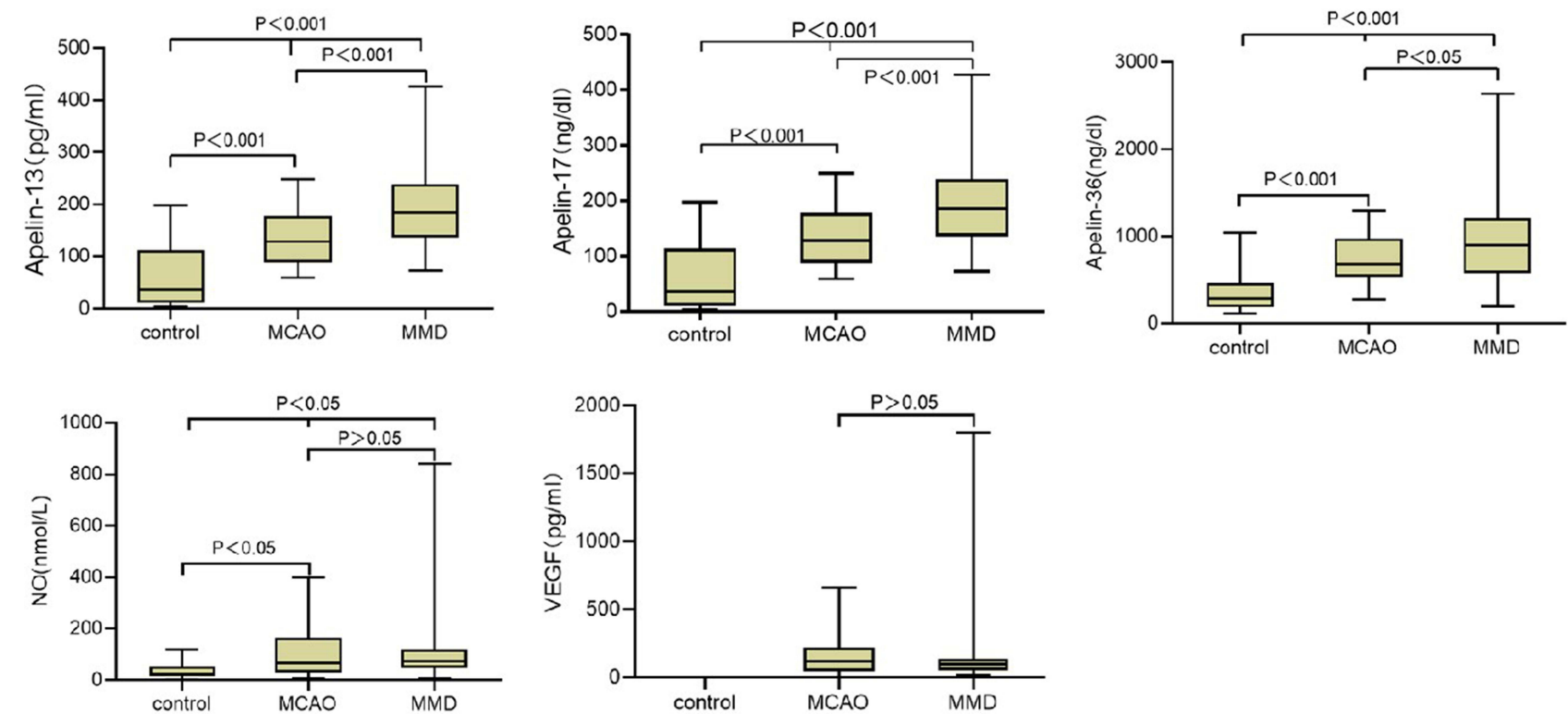

Figure I Plasma apelin-13, apelin- 17, apelin-36, and serum NO and VEGF levels in patients with moyamoya disease (MMD) or middle cerebral artery occlusion and healthy controls.

from MCA occlusion. It may be related to the younger patients with MMD than those with MCA occlusion due to atherosclerosis. Moreover, apelin levels show significant changes in the collateral circulation classification of MMD.

Apelin pro-peptide contains several basic amino acid doublets implicating potential proteolytic cleavage sites for endopeptidases, which would give rise to several bioactive carboxy-terminal (C-terminal) fragments, including apelin-36, apelin-17, and apelin-13. ${ }^{21}$ A previous study has reported that the Apelin/APJ system promotes angiogenesis in MI animal models. ${ }^{22,23}$ For example, Kasai et al found oxygen-induced retinopathy (OIR) in mice treated with Apelin leads to the proliferation of endothelial cells and promoting the process of pathological angiogenesis. ${ }^{24,25}$ Similar reports in critical limb ischemia, tumor, retinal angiogenesis diseases, cirrhosis, obesity, diabetes, and ischemic stroke have been published. However, there are few reports on the relationship between apelin levels and MMD. The relationship between several typical active types of apelin and the course of MMD is still unclear.

Our research revealed that the three kinds of apelin plasma levels (apelin-13, apelin-17, and apelin-36) are significantly higher in patients with MMD than patients with MCA occlusion and healthy individuals. To distinguish MMD and MCA occlusion, binary logistic regression analyses show remarkable differences in plasma apelin-13, apelin-17, and apelin-36 levels. Moreover, it is noteworthy that plasma apelin-13 levels are associated with patients diagnosed as MMD in multivariate logistics regression analysis (confounding factors were regulated). Considering the difference in sample size, this study further conducted a propensity matching, and we found that apelin-13 and apelin-17 levels were still significantly higher in the MMD group after matching, the findings are in line with our hypothesis and similar to previous results of our research group. ${ }^{26}$ A previous study reported that hypoxia-induced apelin expression might provide a new mechanism involved in the adaptive physiological and pathophysiological response of vascular cells to low oxygen level where hypoxic tissues drive the formation of new blood vessels in part by the secretion of apelin, promoting angiogenesis through the proliferation of endothelial cells. ${ }^{27}$ Patients with MMD have a longer incubation period of ischemia caused by the blocked internal carotid artery vessels, which may be related to sufficient vascular compensation. Based on these observations, the higher apelin levels in patients with MMD may explain their greater capacity for angiogenesis and better clinical prognoses and outcomes than patients with stroke caused by occlusion of major intracranial vessels. Based on the binary logistic regression and ROC curve results, we may differentiate MMD from MCA occlusion by detecting apelin plasma levels instead of invasive imaging. Recent studies indicate that the expression of apelin and APLNR 
Table 2 Multivariate Binary Logistics Regression Analysis of MMD and MCA Occlusion Groups, MMD Group Was Used as a Reference

\begin{tabular}{|c|c|c|c|c|}
\hline Parameters & Quartiles & $P$-value & OR & Odds Ratio $(95 \% \mathrm{Cl})$ \\
\hline \multicolumn{2}{|l|}{ Age } & 0.022 & 1.129 & $1.018-1.252$ \\
\hline \multicolumn{2}{|l|}{ Sex (male) } & 0.650 & 1.600 & $0.211-12.130$ \\
\hline \multicolumn{2}{|l|}{ Smoke (yes) } & 0.078 & 0.092 & $0.006-1.313$ \\
\hline \multicolumn{2}{|l|}{ Drunk (yes) } & 0.734 & 1.566 & $0.117-20.866$ \\
\hline \multicolumn{2}{|l|}{ SBP } & 0.846 & 0.996 & $0.962-1.042$ \\
\hline \multirow[t]{4}{*}{ Blood glucose } & $3.83-4.37$ & 0.151 & & \\
\hline & $4.38-4.91$ & 0.026 & 0.037 & $0.002-0.672$ \\
\hline & $4.92-5.46$ & 0.460 & 0.371 & $0.027-5.141$ \\
\hline & $5.47-9.81$ & 0.130 & 0.171 & $0.018-1.679$ \\
\hline \multirow[t]{4}{*}{ Apelin-13 } & $42.02-130.106$ & 0.021 & & \\
\hline & $|30.107-2| 4.97 \mid$ & 0.004 & 61.582 & $3.762-1007.960$ \\
\hline & $2|4.972-3| 4.97 \mid$ & 0.023 & 28.775 & $1.594-519.556$ \\
\hline & $314.972-694.64$ & 0.785 & 1.504 & $0.080-28.14 \mid$ \\
\hline \multirow[t]{4}{*}{ Apelin-17 } & $58.34-121.940$ & 0.411 & & \\
\hline & $|21.94|-172.320$ & 0.479 & 2.542 & $0.192-33.558$ \\
\hline & $|72.32|-231.590$ & 0.394 & 0.269 & $0.013-5.518$ \\
\hline & $231.59|-426.5|$ & 0.582 & 0.465 & $0.030-7.107$ \\
\hline \multirow[t]{4}{*}{ Apelin-36 } & $196.86-577.810$ & 0.106 & & \\
\hline & $577.81 \mathrm{I}-805.640$ & 0.619 & 2.383 & $0.078-72.877$ \\
\hline & $805.64 I-1135.710$ & 0.405 & 4.269 & $0.140-130.222$ \\
\hline & II35.7II-263I.II & 0.035 & 43.659 & $1.310-1454.824$ \\
\hline
\end{tabular}

Notes: The regression model was adjusted by age, sex, smoke, drunk, blood glucose, SBP. We divided continuous variables into four intervals according to the quartile method, the results showed that apelin- 13 in MMD was higher in the MCA occlusion group, and the age of the MCA occlusion group was significantly higher than that of the MMD group.

Abbreviation: SBP, systolic blood pressure.

may be temporally regulated in ischemic stroke. The expression of apelin and APLNR is only increased early after ischemia in the heart. Although glucose deprivation upregulates the expression of apelin and APLNR at early stages, it significantly reduces apelin and APLNR expression at later stages. ${ }^{28,29}$ The indication suggested that elevation of apelin may be a reactive elevation to the ischemic event, not necessarily a persistent phenomenon in the course of the disease.

Among the apelin isoforms, apelin-13 is the most biologically active and most commonly used. ${ }^{30}$ Chronic treatment of apelin-13 increased angiogenesis
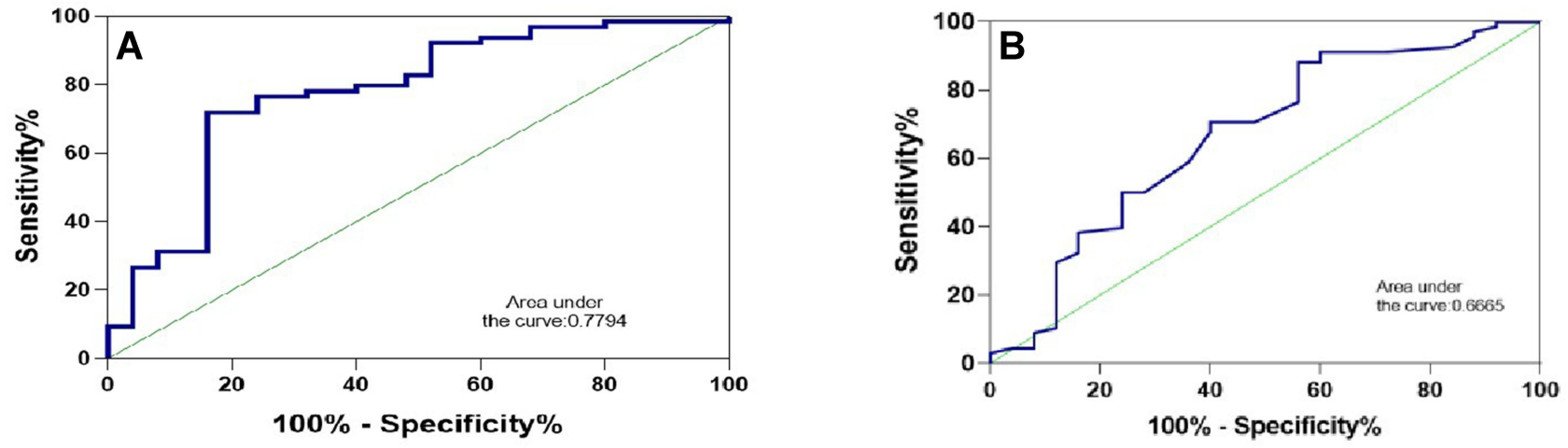

Figure 2 Receiver operating characteristic (ROC) curve analyses of apelin- I3 (A) and age (B) as predictors of moyamoya disease (MMD) from middle cerebral artery occlusion. 


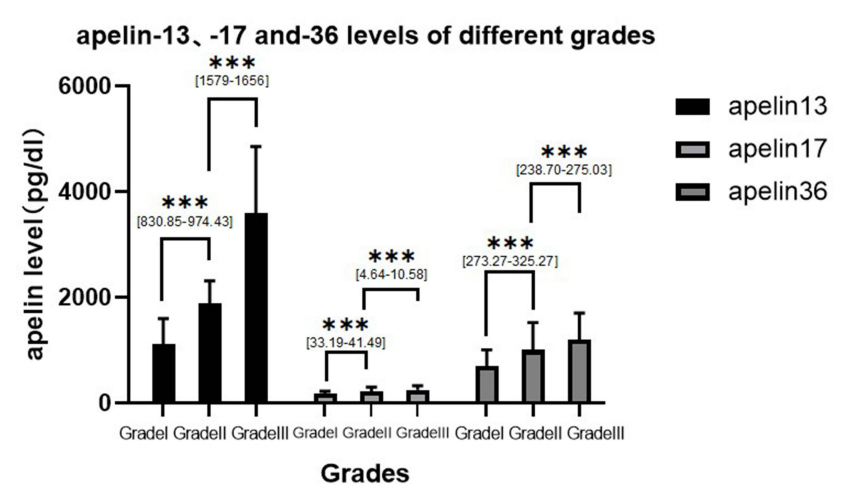

$* * *-\mathrm{P}<0.001$

Figure 3 Linear regression analysis of Apelin levels among different grades in the MMD group. As described above, all MMD patients were divided into three levels, and linear regression was performed on apelin-13, apelin-17, and apelin-36 levels. Through a pairwise comparison between the three groups, the results show that apelin-13, apelin-17, and apelin-36 levels increased as the grades rose without exception (***P<0.00I, $95 \% \mathrm{Cl}$ of $\mathrm{OR}$ are shown inside brackets).

and promoted the LCBF restoration and long-term functional recovery after stroke. The improved blood flow recovery and behavioral recovery are expected to be benefited from neuroprotection and regeneration. Chen et al reported that apelin-13 promotes angiogenesis in focal cerebral ischemic stroke mice, and found that apelin-13 participates in angiogenesis by increasing the expression of VEGF and matrix metalloproteinase-9 (MMP-9). ${ }^{31}$ Regarding other apelin subtypes included in this study, apelin-17 decreased blood pressure and rescued contractile function. ${ }^{32,33}$ Apelin-36 protects against ischemic injury, reduces cerebral infarct volume, and inhibits human immunodeficiency virus (HIV) infection as well as glucose-stimulated insulin secretion. ${ }^{34-36}$ Our previous study highlighted the potential of apelin-12 in the same context of collateralization, there have been relevant studies before. ${ }^{19,37}$ Busch et al demonstrated that apelin-12 activated NO production via $\mathrm{PI} 3 \mathrm{~K} / \mathrm{Akt}$ signaling in human EC. In contrast, apelin-13 additionally activated Erk1/2 phosphorylation and enhanced EC proliferation. ${ }^{38}$ They may also play an important role in collateral circulation, but the mechanisms exist distinction.

Some studies have shown that Apelin improves angiogenesis through the NO-cyclic guanosine monophosphate (cGMP) pathway, and they demonstrated the mechanism in neonatal rats with hyperoxia-induced lung injury. ${ }^{39-41}$ Moreover, other researchers support that the Apelin/APJ system upstream the VEGF in promoting angiogenesis. Apelin promotes angiogenesis by increasing the level of VEGF in post-MI mice: ${ }^{22,42-45}$ however, other studies contrast with those conclusions. ${ }^{46,47}$ In this study, plasma apelin levels in patients with MMD are significantly higher than patients with MCA occlusion and health controls, and the apelin plasma levels show no significant correlation with serum NO and VEGF levels temporarily, this needs to be confirmed by further research.

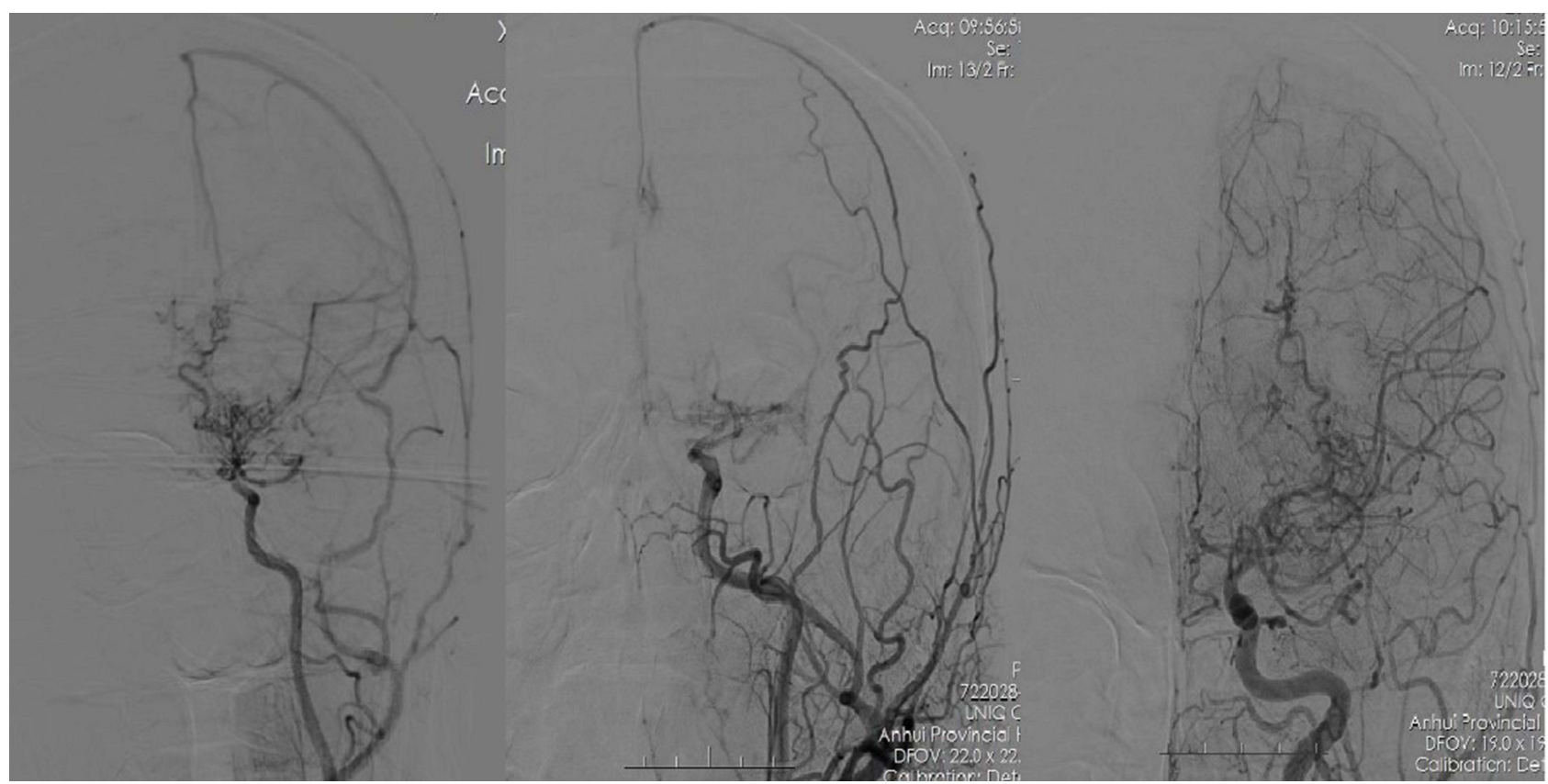

Figure 4 Three DSA images of the included patients with MMD. The compensation gradually improves from left to right (from Grade I to Grade III). 
Meanwhile, our team graded all patients with MMD by digital subtraction angiography with a new grading system. This new MMD collateral grading system correlated well with clinical symptoms, hemodynamic status, and therapeutic prognosis. Moreover, it may facilitate risk stratification and prognosis predictions in MMD patients. It is divided into three levels, $1-4$ points were classified as poor collateral circulation status (Grade I), 5-8 points as general collateral circulation status (Grade II), and 9-12 points as good collateral circulation status (Grade III). ${ }^{20} \mathrm{We}$ found that apelin plasma levels were positively correlated with the classification of MMD for vascular compensation. This finding confirms the speculations of previous researchers, apelin may be related to the vascular compensation of MMD. In addition, previously, we found that apelin-12 may also be a promising candidate for developing clinically therapeutic drugs for ischemic brain disease. ${ }^{22}$ Giving further optimization, novel pro-angiogenesis drugs for serum apelin levels will be investigated, preventing the occurrence and development of ischemic cerebrovascular events and improving the outcomes for patients with MMD.

There are several limitations to this study. Firstly, the sample size is insufficient, especially the number of patients with MCA occlusion; secondly, there was no longitudinal comparative study to confirm; thirdly, not all patients underwent DSA, which may affect our assessment of blood vessels. These are the problems, which are the subject of our future research. Meanwhile, in subsequent studies, we can detect the concentration of apelin levels in the cerebrospinal fluid to see if there are regular changes during blood vessel formation like apelin in the blood, and we can further try drug treatment to improve the prognosis of intracranial aortic occlusion.

\section{Conclusion}

Our study demonstrated that apelin-13 was significantly increased in patients with MMD than patients with MCA occlusion, and there is a truncated value that can differentiate the two clinical diseases. Moreover, plasma apelin13, apelin-17, and apelin-36 levels increase with the improved vascular compensation of MMD, confirming that apelin levels may have a tick relation with the angiogenesis mechanism in MMD and MCA occlusion. Although our sample size is not so satisfactory; apelin-13 might be a promising candidate for determining clinical prognosis in patients with ischemic stroke.

\section{Abbreviations}

MMD, moyamoya disease; MCA, middle cerebral artery; NO, nitric oxide; VEGF, vascular endothelial growth factor; CT, computed tomography; MRI, magnetic resonance imaging; CTA, computed tomographic angiography; DSA, digital subtraction angiography; MRA, magnetic resonance angiographic; ROC, receiver operating characteristic.

\section{Data Sharing Statement}

All data generated or analyzed during this study are included in this published article [and its supplementary information files].

\section{Ethics Approval}

The study was approved by the Department of Neurology and Neurosurgery, The First Affiliated Hospital of University of Science and Technology of China, also named Provincial Hospital Affiliated to Anhui Medical University. The study was approved under expedited review and patient consent was waived as the study design was retrospective. The data confidentiality and compliance with the Declaration of Helsinki were maintained.

\section{Consent for Publication}

We obtained signed consent from the patient for the personal or clinical details to be published in this study.

\section{Author Contributions}

All authors made a significant contribution to the work reported, whether that is in the conception, study design, execution, acquisition of data, analysis and interpretation, or in all these areas; took part in drafting, revising or critically reviewing the article; gave final approval of the version to be published; have agreed on the journal to which the article has been submitted; and agree to be accountable for all aspects of the work.

\section{Funding}

This work was supported by the Key Research and Development Program Projects of Anhui Province in China (No. 1804h08020250). The funder participated in the data acquisition and contributed to review and editing of the article for our study.

\section{Disclosure}

The authors report no conflicts of interest for this work and declare that the research was conducted in the absence of 
any commercial or financial relationships that could be construed as a potential conflict of interest.

\section{References}

1. Apurv G, Anshika T, Moises R, Amoroso Krystal C, Fnu S. Moyamoya disease: a review of current literature. Cureus. 2020. doi:10.7759/cureus.10141

2. Zhang H, Zheng L, Feng L. Epidemiology, diagnosis and treatment of moyamoya disease. Exp Ther Med. 2019. doi:10.3892/etm.2019.7198

3. Burke GM, Burke AM, Sherma AK, Hurley MC, Batjer HH, Bendok BR. Moyamoya disease: a summary. Neurosurg Focus. 2009;26(4):E11. doi:10.3171/2009.1.FOCUS08310

4. Mokin M, Snyder KV, Siddiqui AH, Levy EI, Hopkins LN. Recent endovascular stroke trials and their impact on stroke systems of care. $J$ Am Coll Cardiol. 2016;67(22):2645-2655. doi:10.1016/j. jacc.2015.12.077

5. Liebeskind David S. Stroke: the currency of collateral circulation in acute ischemic stroke. Nat Rev Neurol. 2009;5(12):645-646. doi:10.1038/nrneurol.2009.193

6. Derdeyn Colin P. Moyamoya disease and moyamoya syndrome. N Engl J Med. 2009;361(1). doi:10.1056/NEJMc090780

7. Ayaka S, Takeshi M, Toru H, Yukinori A, Nobuhiro M. Characteristics of cerebral hemodynamics assessed by CT perfusion in moyamoya disease. J Clin Neurosci. 2018;47:183-189. doi:10.1016/j.jocn.2017.09.020

8. Sang-Bok L, Dal-Soo K, Pil-Woo H, Do-Sung Y, Tae-Gyu L, KyoungSuok C. Long-term follow-up results in 142 adult patients with moyamoya disease according to management modality. Acta Neurochir (Wien). 2012;154(7):1179-1187. doi:10.1007/s00701-012-1325-1

9. Rafat N, Beck G, Pena-Tapia PG, Schmiedek P, Vajkoczy P. Increased levels of circulating endothelial progenitor cells in patients with Moyamoya disease. Stroke. 2009;40(2):432-438. doi:10.1161/ STROKEAHA.108.529420

10. Tanaka M, Shimosegawa E, Kajimoto K, et al. Chronic middle cerebral artery occlusion: a hemodynamic and metabolic study with positron-emission tomography. AJNR Am J Neuroradiol. 2008;29 (10):1841-1846. doi:10.3174/ajnr.A1234

11. Lele W, Linxi C, Lanfang L. Apelin/APJ system: a novel promising therapy target for pathological angiogenesis. Clin Chim Acta. 2017;466:78-84. doi:10.1016/j.cca.2016.12.023

12. Chaves-Almagro C, Castan-Laurell I, Dray C, Knauf C, Valet P, Masri B. Apelin receptors: from signaling to antidiabetic strategy. Eur J Pharmacol. 2015;763:149-159. doi:10.1016/j.ejphar.2015.05.017

13. Hiroyasu K, Masaya U, Yoshihiro Y, et al. Spatial and temporal role of the apelin/APJ system in the caliber size regulation of blood vessels during angiogenesis. $\quad E M B O \quad J . \quad 2008 ; 27(3): 522-534 . \quad$ doi:10.1038/sj. emboj.7601982

14. Qin G, Lijing Z, Xing F, et al. Apelin-36, a potent peptide, protects against ischemic brain injury by activating the PI3K/Akt pathway. Neurochem Int. 2013;63(6):535-540. doi:10.1016/j. neuint.2013.09.017

15. Yang Y, Zhang X, Cui H, Zhang C, Zhu C, Li L. Apelin-13 protects the brain against ischemia/reperfusion injury through activating pi3k/ akt and erk1/2 signaling pathways. Neurosci Lett. 2014;568:44-49. doi:10.1016/j.neulet.2014.03.037

16. Nishimoto A, Takeuchi S. Abnormal cerebrovascular network related to the internal carotid arteries. J Neurosurg. 1968;29(3):255-260. doi:10.3171/jns.1968.29.3.0255

17. Adams HP, Bendixen BH, Kappelle LJ, et al. Classification of subtype of acute ischemic stroke. Definitions for use in a multicenter clinical trial. TOAST. Trial of Org 10172 in Acute Stroke Treatment. Stroke. 1993;24(1):35-41. doi:10.1161/01.str.24.1.35
18. Higashida Randall T, Furlan Anthony J, Heidi R, et al. Technology Assessment Committee of the American Society of Interventional and Therapeutic Neuroradiology, Technology Assessment Committee of the Society of Interventional Radiology. Trial design and reporting standards for intra-arterial cerebral thrombolysis for acute ischemic stroke. Stroke. 2003. doi:10.1161/01.STR.0000082721.62796.09

19. Wei H, Wan J, Ye L, Yanghua T, Bing S, Kai W. Prospective evaluation of the diagnostic value of plasma apelin 12 levels for differentiating patients with moyamoya and intracranial atherosclerotic diseases. Sci Rep. 2017. doi:10.1038/s41598-017-05664-8

20. Zhi-Wen L, Cong H, Feng Z, et al. Collateral circulation in moyamoya disease: a new grading system. Stroke. 2019;50 (10):2708-2715. doi:10.1161/STROKEAHA.119.024487

21. Masri B, Knibiehler B, Audigier Y. Apelin signalling: a promising pathway from cloning to pharmacology. Cell Signal. 2005;17 (4):415-426. doi:10.1016/j.cellsig.2004.09.018

22. Zuocheng W, McKinnie Shaun MK, Patel Vaibhav B, et al. Loss of Apelin exacerbates myocardial infarction adverse remodeling and ischemia-reperfusion injury: therapeutic potential of synthetic Apelin analogues. J Am Heart Assoc. 2013;2(4). doi:10.1161/JAHA.113.000249

23. Shohei K, Hiroyuki N, Minoru F, et al. Myeloid cell-derived LRG attenuates adverse cardiac remodelling after myocardial infarction. Cardiovasc Res. 2016;109(2):272-282. doi:10.1093/cvr/cvv273

24. Kasai A, Ishimaru Y, Higashino K, et al. Inhibition of apelin expression switches endothelial cells from proliferative to mature state in pathological retinal angiogenesis. Angiogenesis. 2013;16(3):723-734. doi:10.1007/s10456-013-9349-6

25. Atsushi K, Yuki I, Toshihiko K, et al. Apelin is a crucial factor for hypoxia-induced retinal angiogenesis. Arterioscler Thromb Vasc Biol. 2010;30(11):2182-2187. doi:10.1161/ATVBAHA.110.209775

26. Wan J, Wei H, Ye L, et al. Contribution of Apelin-17 to collateral circulation following cerebral ischemic stroke. Transl Stroke Res. 2019;10(3):298-307. doi:10.1007/s12975-018-0638-7

27. Mélanie E, Géraldine S, Mariana C, et al. Hypoxia-induced apelin expression regulates endothelial cell proliferation and regenerative angiogenesis. Circ Res. 2008;103(4):432-440. doi:10.1161/ CIRCRESAHA.108.179333

28. Rastaldo R, Cappello S, Folino A, Losano G. Effect of apelin-apelin receptor system in post ischaemic myocardial protection: a pharmacological postconditioning tool? Antioxid Redox Signal. 2011;14(5):909-922. PMID: 20615122. doi:10.1089/ars.2010.3355

29. Zhang Z, Yu B, Tao GZ. Apelin protects against cardiomyocyte apoptosis induced by glucose deprivation. Chin Med J (Engl). 2009;122(19):2360. PMID: 20079140.

30. Jun C, Xuling L, Zhen H, Linxi C. Apelin/APJ system: a potential therapeutic target for endothelial dysfunction-related diseases. $J$ Cell Physiol. 2019;234(8):12149-12160. doi:10.1002/jcp.27942

31. Chen D, Lee J, Gu X, Ling W, Ping YS. Intranasal delivery of Apelin-13 is neuroprotective and promotes angiogenesis after ischemic stroke in mice. ASN Neuro. 2015;7(5). doi:10.1177/1759091415605114

32. Wang W, McKinnie Shaun MK, Maikel F, et al. Angiotensinconverting Enzyme 2 metabolizes and partially inactivates Pyr-Apelin-13 and Apelin-17: physiological effects in the cardiovascular system. Hypertension. 2016;68(2):365-377. doi:10.1161/ HYPERTENSIONAHA.115.06892

33. Habata Y, Fujii R, Hosoya M, et al. Apelin, the natural ligand of the orphan receptor APJ, is abundantly secreted in the colostrum. Biochim Biophys Acta. 1999. doi:10.1016/s0167-4889(99).

34. Kenji S, Yuta M, Shohei S, et al. Apelin-36 is protective against N-methylD-aspartic-acid-induced retinal ganglion cell death in the mice. Eur J Pharmacol. 2016;791:213-220. doi:10.1016/j.ejphar.2016.08.036

35. Kleinz Matthias J, Davenport Anthony P. Emerging roles of apelin in biology and medicine. Pharmacol Ther. 2005;107(2):198-211. doi:10.1016/j.pharmthera.2005.04.001 
36. Maria SW, Caroline M, Ahrén B. The APJ receptor is expressed in pancreatic islets and its ligand, apelin, inhibits insulin secretion in mice. Regul Pept. 2005;131(1-3):12-17. doi:10.1016/j.regpep.2 005.05 .004

37. Kiyoshige T, Hiromi I, Misako H, Koji Y, Shoki R. Distribution of c-Fos immunoreactive neurons in the brain after intraperitoneal injection of apelin-12 in Wistar rats. Neurosci Lett. 2008. doi:10.1016/j. neulet.2007.11.048

38. Busch R, Anne S, Malte P, et al. Regulation of the endothelial apelin/ APJ system by hemodynamic fluid flow. Cell Signal. 2015;27 (7):1286-1296. doi:10.1016/j.cellsig.2015.03.011

39. de Visser Yvonne P, Walther Frans J, El Houari L, et al. Apelin attenuates hyperoxic lung and heart injury in neonatal rats. $\mathrm{Am}$ $J$ Respir Crit Care Med. 2010. doi:10.1164/rccm.200909-1361OC

40. Odette P, Mariana C, Mélanie E, Kevin M, Sophie N, Florent S. Inhibition of apelin expression by BMP signaling in endothelial cells. Am J Physiol Cell Physiol. 2012. doi:10.1152/ajpcell.00168.2012

41. Nickel Nils P, Edda S, Mingxia G, et al. Elafin reverses pulmonary hypertension via Caveolin-1-dependent bone morphogenetic protein signaling. Am J Respir Crit Care Med. 2012. doi:10.1164/ rccm.201412-22910C

42. Lanfang L, Heng Z, Jian-Xiong C. Apelin-13 increases myocardial progenitor cells and improves repair postmyocardial infarction. $\mathrm{Am}$ J Physiol Heart Circ Physiol. 2012;303(5):H605-18. doi:10.1152/ ajpheart.00366.2012
43. Hou X, Zeng H, He X, Chen J-X. Sirt3 is essential for apelin-induced angiogenesis in post-myocardial infarction of diabetes. $\mathrm{J}$ Cell $\mathrm{Mol}$ Med. 2015;19(1):53-61. doi:10.1111/jcmm.12453

44. Yaser A, Mahdieh F, Alireza I, et al. Post-infarct treatment with [Pyr (1)]apelin-13 improves myocardial function by increasing neovascularization and overexpression of angiogenic growth factors in rats. Eur J Pharmacol. 2015. doi:10.1016/j.ejphar.2015.04.034

45. Zhang B-H, Guo C-X, Wang H-X, et al. Cardioprotective effects of adipokine apelin on myocardial infarction. Heart and Vessels. 2014;29(5):679-689. doi:10.1007/s00380-013-0425-Z

46. Kälin Roland E, Kretz Martin P, Meyer Andrea M, Andreas K, Heppner Frank L, Brändli André W. Paracrine and autocrine mechanisms of apelin signaling govern embryonic and tumor angiogenesis. Dev Biol. 2007;305(2):599-614. doi:10.1016/j.ydbio.2007.03.004

47. Chikako H, Atsushi K, Fumi G, et al. Laser-induced choroidal neovascularization in mice attenuated by deficiency in the apelin-APJ system. Invest Ophthalmol Vis Sci. 2013;54(6):4321-4329. doi:10.1167/iovs.13-11611
International Journal of General Medicine

\section{Publish your work in this journal}

The International Journal of General Medicine is an international, peer-reviewed open-access journal that focuses on general and internal medicine, pathogenesis, epidemiology, diagnosis, monitoring and treatment protocols. The journal is characterized by the rapid reporting of reviews, original research and clinical studies

\section{Dovepress}

across all disease areas. The manuscript management system is completely online and includes a very quick and fair peer-review system, which is all easy to use. Visit http://www.dovepress.com/ testimonials.php to read real quotes from published authors. 